\title{
Atuação da equipe de enfermagem no parto humanizado e seus benefícios
} diante o parto cesárea

\author{
Mirian Aparecida Gomes ${ }^{1}$, Chennyfer Dobbins Abi Rached ${ }^{2}$
}

1 Graduanda de Enfermagem. Universidade Nove de Julho - UNINOVE. São Paulo - Brasil

- Email: São Paulo - Brasil - email: miheron7@gmail.com

2 Doutora em Saúde Coletiva; Mestre em Economia da Saúde pela Universidade Federal de São Paulo (UNIFESP); Docente do Programa de Mestrado Profissional em Gestão em Sistemas de Saúde - Universidade Nove de Julho - UNINOVE. São Paulo - Brasil email: chennyferr@yahoo.com.br ORCID: https://orcid.org/0000-0002-4499-3716

\begin{abstract}
RESUMO
Introdução: O parto é um processo natural e fisiológico, porém após a institucionalização desse processo, o parto tornou-se mecanizado. A definição acerca da via de parto sofre influência de diversos fatores, dentre os quais se destacam: riscos e benefícios, possíveis complicações e repercussões futuras. Objetivos: Identificar os malefícios do parto cesárea junto às suas possíveis complicações quando comparados ao parto humanizado; Descrever a atuação da equipe de enfermagem no parto humanizado. Método: Pesquisa bibliográfica em bases de dados LILACS e SCIELO, e na biblioteca eletrônica Biblioteca Virtual em Saúde (BVS) de artigos de periódicos nacionais, sendo textos de língua portuguesa, disponíveis gratuítos na íntegra e a partir do ano de 2013. Desenvolvimento: A cesariana sem indicação clínica quando comparada ao parto vaginal está relacionada com maior morbidade materna, como a necessidade de transfusão sanguínea, histerectomia, internação em UTI e piores desfechos neonatais, enquanto o parto humanizado passa por um modelo assistencial que proporciona a mulher benefícios físicos e psicológicos. O enfermeiro ao elaborar intervenções voltadas para as reais necessidades das puérperas, qualifica o cuidado prestado com a contribuição de forma decisiva. Considerações Finais: O parto normal é um desafio para as mulheres, porém benéfico em relação à cesárea, que por sua vez, apesar de ser mais rápida e cômoda, pode acarretar em diversar complicações materno-fetal.
\end{abstract}

Palavras-chave: Parto Humanizado; Cuidados de Enfermagem; Cesárea.

\begin{abstract}
Introduction: Childbirth is a natural and physiological process, but after the institutionalization of this process, childbirth has become mechanized. The definition about the way of delivery is influenced by several factors, among which the following stand out: risks and benefits, possible complications and future repercussions. Objectives: To identify the harmful effects of cesarean section with its possible complications when compared to humanized delivery; To describe the performance of the nursing team in humanized childbirth. Method: Bibliographic research in LILACS and SCIELO databases, and in the electronic library Virtual Health Library (VHL) of national periodical articles, being Portuguese-language texts, available free of charge in full and from 2013. Development: A cesarean section without clinical indication when compared to vaginal delivery is related to increased maternal morbidity, such as the need for blood transfusion, hysterectomy, ICU admission and worse neonatal outcomes, whereas humanized delivery goes through a care model that provides women with physical and psychological benefits. Nurses, when elaborating interventions aimed at the real needs of puerperal women, qualify the care provided with the contribution in a decisive way. Final Considerations: Normal delivery is a challenge for women, but beneficial in relation to cesarean section, which, in turn, despite being quicker and more comfortable, can lead to a variety of maternal-fetal complications. Key words: Humanized Delivery; Nursing care; Cesarean section.
\end{abstract}




\section{INTRODUÇÃO}

O parto é um processo natural e fisiológico que desde os primórdios era realizado de maneira instintiva pela mulher e auxiliado por pessoas que tinham certo conhecimento sobre o nascimento, porém após a institucionalização desse processo, o parto tornou-se mecanizado e tecnicista protagonizado geralmente pelo profissional médico que entende o parto como doença e que realiza procedimentos intervencionistas dispensáveis para tratá-lo. Assim, o profissional deixa de lado o contexto psicossocial que envolve esse momento, desumanizando a mulher e retirando todo o seu poder e vontade sobre esse procedimento ${ }^{1}$.

A OMS preconiza que o objetivo da assistência ao nascimento é promover o mínimo possível de intervenção, com segurança, para obter uma mãe e uma criança saudáveis. Suas recomendações para a assistência ao parto consistem na mudança de um paradigma, entre elas registram-se: o resgate da valorização da fisiologia do parto; o incentivo de uma relação de harmonia entre os avanços tecnológicos e a qualidade das relações humanas; além de destacar o respeito aos direitos de cidadania ${ }^{3,4}$.

Vale destacar que o parto cesáreo é definido como a extração do feto através de uma incisão na parede abdominal e na parede uterina. Em geral, a cesariana é realizada quando o trabalho de parto está contra indicado ou quando não é provável que o parto vaginal seja realizado com segurança ${ }^{1}$.

Apesar da contribuição dessa intervenção para uma melhor assistência à saúde, contribuindo para a queda da mortalidade materna e neonatal, é importante que sua indicação seja criteriosa, pois sua realização sem justificativa clínica pode agregar riscos para a mãe e a criança, sem um benefício claro ${ }^{4}$.

$\mathrm{O}$ parto operatório quando começou ser realizado proporcionava taxas elevadas de complicações maternas e fetais, além de aumentar o número de óbitos. Porém com o passar dos tempos com o aperfeiçoamento de novas técnicas e de maiores estudos sobre o assunto esse patamar se inverteu. Sendo que atualmente o índice de cesariana é muito grande, recorrente talvez do diagnóstico mais preciso de sofrimento fetal e das cesáreas eletivas ${ }^{5}$.

As taxas de cesariana aumentaram significativamente nas últimas décadas. No ano de 2008, 6.2 milhões de cesáreas desnecessárias foram realizadas no mundo; dessas, China e Brasil representaram quase $50 \%$ do total de cesarianas sem indicação médica ${ }^{6}$.

No Brasil, a Organização Mundial de Saúde (OMS) considera epidêmica a elevação das taxas de cesárea, com valores que superam os $15 \%$ preconizados ${ }^{2,4}$. Os Indicadores e Dados Básicos para a Saúde do Brasil fornecem, em seus índices de cobertura, a proporção de partos cesáreos, cujos valores alcançaram o patamar de 52,34\%, no ano de $2010^{2}$. 
Esse aumento no número de cesáreas em todo o mundo está relacionado à melhoria de acesso de mulheres a esse procedimento em caso de necessidade, mas também à realização indiscriminada sem indicação médica. Com isso, há grandes esforços recentes para diminuir essas taxas, ao mesmo tempo em que são incorporadas as preferências obstétricas das mulheres ${ }^{6}$.

A utilização indiscriminada das cesarianas muito pouco tem a ver com a necessidade clínica da gestante e ou do recém-nascido, mas com a vontade da mãe e do médico e com o nível socioeconômico da família. Via de regra, este procedimento é realizado entre àquelas com menor risco de complicações durante a gravidez e o parto ${ }^{7}$.

A definição acerca da via de parto sofre influência de diversos fatores, dentre os quais se destacam: riscos e benefícios, possíveis complicações e repercussões futuras ${ }^{8}$. Apesar da importância inegável desse procedimento, gestantes e profissionais de saúde precisam conhecer os riscos maternos associados às diferentes vias de parto, utilizando as melhores evidências ${ }^{6}$.

De modo geral, a indicação de uma cesariana é feita mediante o diagnóstico de intercorrências durante a gestação ou trabalho de parto, havendo possibilidade limitada de agendamento de cesariana a pedido da mulher'.

Cesáreas realizadas de forma adequada e seguindo uma indicação médica precisa são procedimentos potencialmente salvadores de vidas. Entretanto, por um lado, o fornecimento de cesarianas seguras e oportunas ainda é um grande desafio em países com grande mortalidade materna, onde são insuficientes; por outro lado, seu excesso em determinadas regiões faz com que o desafio consista em minimizar a realização de cesarianas sem indicação clínica $^{6}$.

Pensando em uma melhor assistência às parturientes, a Organização Mundial de Saúde (OMS) e o Programa de Humanização no Pré-Natal e Nascimento (PHPN) estabelecem um novo modelo de assistência obstétrica, na qual caracteriza a mulher como protagonista do processo de parir, valorizando uma assistência mais humanizada que proporciona um atendimento de qualidade, articulando um acolhimento que já começa logo no pré-natal e vai até o nascimento, com a finalidade de garantir uma assistência obstétrica integral à mulher e preservar seus direitos de escolha, sendo o parto realizado com o mínimo de intervenções possíveis respeitando a fisiologia da mulher e sua autonomia ${ }^{10}$.

\section{JUSTIFICATIVA E PROBLEMA}


Escolhi esse tema devido as mulheres, muitas vezes, serem induzidas pelo seus planos de saúde unicamente preocupados com o que vão faturar sem se preocupar com os malefícios a mãe e ao bebê. Pois cada vez mais as mulheres optam pela cesariana a fim de evitar a dor das contrações esquecendo de suas complicações o parto normal e um procedimento físiológico trazendo muitos benefícios e pode evitar complicações já que não a necessidade de intervenção cirúrgica.

Diante o exposto, surgiram as problemáticas: quais os benefícios do parto normal humanizado diante o parto cesárea? Qual a atuação da equipe de enfermagem mediante o parto humanizado?

\section{OBJETIVOS}

\subsection{OBJETIVOS GERAIS}

- Identificar artigos científicos nacionais que abordem a assistência de enfermagem no parto humanizado.

\subsection{OBJETIVOS ESPECÍFICOS}

- Descrever a atuação da equipe de enfermagem no parto humanizado;

- Identificar os malefícios do parto cesárea junto às suas possíveis complicações quando comparados ao parto humanizado.

\section{MÉTODO}

Para realização do estudo, foi utilizado o método de pesquisa bibliográfica, o qual consiste no exame da bibliografia, para o levantamento e análise do que já foi produzido sobre o assunto que é tema da pesquisa científica. Dessa forma, foi utilizado o levantamento bibliográfico e, em seguida, a coleta de informações, dados, fatos e informações contidas na bibliografia selecionada.

O levantamento foi realizado em bases de dados LILACS - Literatura Latino-Americana em Ciências de Saúde e SCIELO - Scientific Eletronic Library Online, no sítio da BVS Biblioteca Virtual em Saúde (site composto por fontes de informação em ciências da saúde), utilizando-se como palavras-chave os termos: parto humanizado, cuidados de enfermagem, cesárea.

Foi feito uma leitura exploratória e seletiva, para verificar se existem, ou não, informações a respeito do tema proposto e coerência com o objetivo do estudo. 
De acordo com esta leitura, foram encontrados cerca de 55 (cinquenta e cinco) artigos e selecionados somente 15 (quinze) artigos que abordaram o tema da pesquisa e que estavam publicados entre os anos de 2013 a 2018, que estavam disponíveis na íntegra gratuitamente e em português.

Excluiu-se, portanto, os artigos publicados fora deste período e aqueles que não abordaram sobre a temática em questão.

A partir dos artigos selecionados, foi realizado uma leitura crítica e interpretativa com a necessária imparcialidade e objetividade, no qual foram relacionadas às informações e idéias dos autores com o objetivo do estudo.

\section{DESENVOLVIMENTO}

\subsection{PARTO NORMAL HUMANIZADO E ENFERMAGEM}

As recomendações da OMS, sobre a assistência ao parto normal, afirmam: "deve existir uma razão válida, para interferir no processo natural", contudo existem posturas contrastantes na assistência obstétrica brasileira ${ }^{2}$. É importante haver esforços para desestimular tais práticas inseguras as quais colocam a mulher em risco e não garante o seu bem-estar ${ }^{1}$.

O governo brasileiro preconiza uma assistência humanizada à mulher no processo de nascimento e para contribuir com este aspecto, estudos apontam a necessidade de acesso das mulheres a um conhecimento que tenha embasamento científico, que forneça à mulher maior segurança, participação no controle decisório e autonomia no momento do nascimento; um embasamento teórico que contribua para a reflexão da assistência recebida ainda que, nas relações entre os profissionais de saúde e as parturientes, estejam envolvidos aspectos complexos, que vão além do poder de argumentação da mulher ${ }^{2}$.

O conceito de atenção humanizada é amplo e envolve um conjunto de conhecimentos, práticas e atitudes que visam à promoção do parto e do nascimento saudável e à prevenção da morbimortalidade materna e perinatal. Faz- se necessário uma relação de confiança, que foque às necessidades e anseios da parturiente, além de permitir uma concepção segura ${ }^{1}$.

É de extrema importância à humanização do parto por parte dos profissionais, fazendo com que estes não se detenham apenas as questões ligadas à saúde materno-fetal, mas que deem atenção também ao estado emocional e psicológico enfrentado pelas parturientes, fornecendo suporte a estas nos momentos de dor, mantendo a privacidade da mulher durante o parto e respeitando sua escolha pela via de parturição ${ }^{5}$.

A humanização do parto não tem o enfoque somente técnico, mas também deve ser associado ao respeito dos direitos da paciente, o que incitou os hospitais da rede pública a adotarem 
medidas humanizadas, que envolvia a participação da mulher ativamente nas decisões, como escolher a posição para parir, utilizar água morna como mecanismo não farmacológico de alívio à dor, ter liberdade para movimentar-se, fazer exercícios e ser assistida por alguns profissionais de saúde treinados para oferecer as orientações que se fizerem necessárias ${ }^{10}$.

A enfermagem obstétrica tem desempenhado um papel importante nos cuidados humanísticos às mulheres, favorecendo a fisiologia do parto e introduzindo tecnologias que proporcionam o cuidado e conforto à mulher, inserindo em seu atendimento habilidades e competências profissionais, como também a utilização de técnicas e conhecimentos múltiplos e complexos, que possam acompanhar o processo parturitivo, o que resulta na promoção da saúde da mulher e do bebê. Tal profissional tem um cuidado diferenciado, uma formação éticohumanística, com qualidades de afeto, respeito e segurança para com o corpo e o lado afetivo da mulher, com uma assistência que vai muito além de técnicas, práticas relacionais, que possibilitam diálogo entre profissionais e usuários, estabelecendo, assim, uma conduta de acolhimento. Um profissional de olhar holístico ${ }^{10}$.

A relação profissional-paciente necessita de escuta não só como um ato de boa vontade, mas como um imprescindível recurso para o diagnóstico e a adesão terapêutica ${ }^{11,12}$. Onde o respeito, solidariedade, apoio, orientação e incentivo são fatores que demonstram o cuidado e importância da assistência humanizada desse profissional ${ }^{12}$.

O parto humanizado passa por um modelo assistencial que proporciona a mulher benefícios físicos e psicológicos, deixando-a como centro de todo o processo, controle das ações e decisões a serem tomadas por ela, mas com acompanhamento e segurança oferecidas pelo profissional de saúde em especial o enfermeiro participante do momento vivido pela parturiente $^{11}$.

Os profissionais de saúde devem orientar as mulheres com informações claras, visando a otimizar o bem-estar do binômio mãe e filho, esclarecendo sobre os riscos e benefícios de cada via de parto em diversas situações. A escolha da mãe, quando ela inicia essa conversa, sem oferta por parte do médico, desde que esclarecida e mantendo a segurança do feto, deve ser soberana, respeitando sua autonomia 6 .

Segundo a OMS, o parto humanizado tem início no pré-natal com o aconselhamento e orientações, respeitando a individualidade da parturiente, seus desejos e, durante o trabalho de parto, dar liberdade de escolha quanto à posição mais apropriada e agradável para parir ${ }^{1}$.

A ação educativa durante o pré-natal é de extrema relevância, pois é nessa ocasião que ocorre a preparação tanto física como psicológica da mulher para o ato da maternidade. A qualidade do pré-natal influencia na cadeia de crenças e opiniões sobre as vias de parto e, 
consequentemente, sobre a escolha final. O profissional de saúde, no papel de educador, tem que estar atento a esta assistência ${ }^{13}$.

O papel do profissional, além de fornecer as orientações em geral tais como saber lidar com a dor e com o desconforto, é também orientar a fazer adequadamente os exercícios respiratórios; estimulá-la a fazer uso do banho de chuveiro, à deambulação, a praticar exercícios de agachar e levantar, exercícios com a bola, aplicar-lhe massagem, enfim, fazer uso de recursos para tornar o processo menos doloroso e fazer com que a mulher fique mais relaxada e colaborativa, sendo que os métodos não farmacológicos oferecidos durante o trabalho de parto são de suma importância para as parturientes ajudando-as nos momentos de tensão e dor ${ }^{10}$.

É imprescindível a participação do enfermeiro obstetra/obstetriz na atenção ao ciclo gravídico puerperal, pois este profissional desempenha um papel estratégico no processo educativo, além de assistir a mulher com qualidade e de forma mais humanizada ${ }^{13}$.

A qualificação profissional de enfermeiros, para prestar um serviço humanizado de qualidade e capacita-as a atuarem em diferentes momentos do ciclo gravídico: pré-natal, parto, nascimento e pós-parto. Os enfermeiros que podem realizar o parto normal são: enfermeiro e o obstetriz/enfermeiro obstétrico, assim como a parteira titulada no Brasil até 1959. Evidências mostram que modelos de assistência envolvendo esses profissionais associam-se a menores taxas de intervenções e em maior satisfação das mulheres ${ }^{10,13}$.

A prática assistencial da maioria dos enfermeiros obstetras é voltada à valorização da mulher, fortalecendo sua parturição, mediante apoio psicológico, respeito ao seu tempo, com boa comunicação e compreensão ${ }^{10}$.

Percebe-se que ao informar à parturiente sobre os diversos procedimentos aos quais será submetida, oferecer um ambiente acolhedor e confortável e esclarecer suas dúvidas são ações simples e que requerem a boa vontade do profissional de saúde ${ }^{1}$.

$\mathrm{Na}$ admissão, deve-se respeitar a privacidade da mulher e a escolha do seu acompanhante. Durante o trabalho de parto, líquidos devem ser oferecidos, além de um suporte emocional, oferecendo informações sobre os procedimentos realizados e encorajar a posição não deitada, deve-se também dar liberdade de posição e movimento à parturiente. $\mathrm{O}$ controle da dor deve ser feito por meios não invasivos e não farmacológicos, como técnicas de relaxamento, massagens, entre outros ${ }^{1,14}$.

O enfermeiro ao elaborar intervenções voltadas para as reais necessidades das puérperas, qualifica o cuidado prestado com a contribuição de forma decisiva para a prevenção e redução das taxas de infecção puerperal. Assim, o puerpério é um período de risco, os quais tornam 
essenciais os cuidados de enfermagem qualificados que tenham como base a prevenção de complicações ${ }^{14}$.

O enfermeiro deve estar alerta ás queixas e outras manifestações que possam indicar algum tipo de intercorrências, informando a gestante sobre a evolução do trabalho de parto e ensinando-lhe as condutas a serem tomadas durante período de dilatação ${ }^{11}$.

É de extrema importância a capacitação e atuação da equipe de enfermagem, sendo dever do profissional orientar a parturiente e família sobre o andamento do parto, também saber controlar e planejar estratégias para que tais dificuldades sejam superadas, para minimizar traumas e sofrimento para ambas as partes, podendo passar por complicações irreversíveis. A humanização da assistência é percebida pela equipe de enfermagem obstétrica como a promoção do cuidado integral de caráter não invasivo, um vínculo de confiança entre paciente e profissional, no qual ambos passam a compartilhar os sentimentos, planejamentos, e as decisões dos seus cuidados prestados ${ }^{10}$.

É necessário um esforço muito grande para sensibilizar e motivar os profissionais de saúde da atenção básica e, é preciso também fornecer instrumentos para que desenvolvam o trabalho com as gestantes. Além dos aspectos técnicos o preparo para o parto envolve uma abordagem onde prioriza o acolhimento da mulher e seu companheiro no serviço de saúde, incluindo o fornecimento de todas as informações desde onde e como o nascimento deverá ocorrer, o preparo físico e psíquico da mulher, idealmente uma visita à maternidade para conhecer suas instalações físicas, o pessoal e os procedimentos rotineiros, entre outros ${ }^{11}$.

Contudo, assistência humanizada no trabalho de parto e parto, proporciona muitos benefícios a parturiente trazendo conforto, bem estar e até mudanças nos conceitos e pré-conceitos em relação ao ato de parturição. Permitindo assim que ela, a parturiente seja protagonista deste momento tão esperado, participando intimamente e ativamente das decisões sobre seu próprio cuidado $^{1,11}$.

\subsection{BENEFÍCIOS PARTO HUMANIZADO}

O parto normal é a maneira mais segura e saudável de ter filhos e, por isso, deve ser estimulado e realizado através de uma assistência humanizada, segura e de qualidade. A cesariana, só deve ser realizada quando for realmente necessária para proteger a gestante e o bebê. Sendo assim, a gestante tem o direito de ser informada sobre os motivos que a impedem de optar pelo parto normal ${ }^{1}$. 
Atualmente, há uma demanda crescente do parto normal no SUS e na Saúde Suplementar, por meio de práticas que aumentam a satisfação das parturientes, uso restrito de procedimentos invasivos, inserção da enfermagem obstétrica, criação de equipes de plantão e, ainda, por intermédio das publicações de diretrizes que qualificam a atenção ao parto e nascimento no país ${ }^{15}$.

Entre os fatores mais referidos para a opção pelo parto vaginal verificaram-se a recuperação menos demorada, a qual não proporciona dor no pós-parto, sensação de dor apenas ao momento do nascimento, o parto vaginal é próprio da fisiologia humana, desencadeia menos danos à mãe e ao bebê, não há incisão cirúrgica no abdômen que possa oferecer cicatrização dificultada, risco para a infecção ${ }^{5}$ e o procedimento parto vaginal apresenta menor custo do que a cesariana eletiva para gestantes de risco habitual, independentemente da paridade ${ }^{15}$.

Os aspectos positivos do parto normal são: o parto é mais simples, rápida, fácil e tranquila. A recuperação é melhor, a mulher sente-se a mesma depois do parto normal, com independência para caminhar, realizar cuidados de higiene pessoal e atividades domésticas, cuidar do bebê, além de uma recuperação mais rápida do seu próprio corpo, pois o parto normal possibilita adotar uma postura ativa, movimentar o seu corpo, receber massagens, realizar o banho terapêutico, exercícios com a bola e o cavalinho².

Ressalta-se que na realização do parto normal humanizado, é essencial que cuidados não farmacológicos de alívio da dor sejam explorados; a equipe de enfermagem tem um papel fundamental na realização desses cuidados, proporcionando à parturiente alívio da dor através de medidas simples como liberdade de adotar posturas e posições variadas, exercícios que facilitam o trabalho de parto, deambulação, respiração ritmada e ofegante, banhos de chuveiro e de imersão, toque e massagem, dando à mulher a oportunidade de ter uma boa vivência deste momento especial que é a chegada do filho ${ }^{1}$.

O medo do procedimento cirúrgico para retirada do bebê, o pavor de sofrer complicações, o temor de sentir os efeitos colaterais decorrentes da anestesia mostraram-se favorável ao parto normal ${ }^{5,13}$.

Sendo assim, o parto normal é ativo e mais saudável por ser natural, tornando a parturiente protagonista no ato, o que não é percebido no parto cesáreo, pois, nele, a mulher assume a postura passiva, perdendo em partes o sentido do protagonismo ${ }^{13}$.

\subsection{MALEFÍCIOS DA CESÁRIA}

A elevada percentagem de partos cesáreos representa um grande desafio para a política de saúde, considerando os riscos desnecessários tanto para a mãe quanto para a criança, além da 
sua associação com a mortalidade materna e os custos adicionais para o sistema de saúde; especialmente quando a redução da mortalidade materna está associada à atenção qualificada ao parto $^{13}$.

O principal motivo pelas mulheres escolherem o parto cesárea, é alegando evitar dor, lacerações ou por tocofobia, que se refere ao medo irracional do parto vaginal. A ocorrência deste tipo de cesariana sem indicação médica vem aumentando em todo o mundo, e isto viola os princípios da boa prática médica, submete a paciente a riscos desnecessários e aumenta o gasto do setor saúde. Entre $6 \%$ e $17 \%$ das cesarianas em diferentes países ocorrem por solicitação materna ${ }^{7}$.

O modelo de assistência ao parto, no Brasil, é caracterizado por excesso de intervenção, o que tem contribuído para o aumento de cesáreas e a morbimortalidade materna e infantil. A razão da mortalidade materna relacionada com os nascimentos cirúrgicos continua sendo um dos indicadores que mais contribuem para esse aumento ${ }^{13}$.

A cesárea é vista como um procedimento complicado, associado a eventos negativos desde o princípio, bem como um resultado das complicações da gravidez. Alguns sentimentos negativos são identificados, entre os quais está o temor, o nervosismo, o receio de complicações, a aversão e o trauma do procedimento ${ }^{2}$.

O parto no ambiente hospitalar tem se caracterizado como evento cirúrgico e desumano, deixando de ser privado, íntimo e feminino, o que eleva a taxa de infecção puerperal. Daí a necessidade da redução de procedimentos hospitalares desnecessários à mulher no período gravídico puerperal ${ }^{14}$.

Quando se define pela realização de uma cesariana, a puérperas submetida a esse procedimento cirúrgico requer cuidados de pós-operatório, bem como deve realizar cuidados essenciais ao recém-nascido e estar devidamente preparada para a amamentação. A dor presente após a cesárea dificulta uma recuperação imediata, fazendo com isso que haja um retardo no contato da mãe com o recém-nascido. Dessa forma, as queixas álgicas no pósoperatório de cesariana podem representar um obstáculo ao bom posicionamento para a amamentação, para o autocuidado, para os cuidados com o recém-nascido e, por fim, para realizar atividades cotidianas, tais como: sentar e levantar, caminhar, realizar higiene íntima entre outras ${ }^{8}$.

A cesárea por se tratar de um procedimento cirúrgico e invasivo, quando comparado ao parto normal, que é um processo natural e fisiológico, apresenta maior número de complicações. $\mathrm{O}$ problema dessa prática é a sua associação com complicações maternas e fetais, como, por exemplo: acidentes anestésicos, hemorragias, lesões vesicais e intestinais, alongamento da incisão miometrial, embolia amniótica e infecção puerperal ${ }^{14}$. 
A cesariana eletiva sem indicação clínica quando comparada ao parto vaginal está relacionada com maior morbidade materna, como a necessidade de transfusão sanguínea, histerectomia, internação em UTI e piores desfechos neonatais, como o aumento de internação em UTI e mortalidade neonatal. Em termos de indicadores de utilização de serviços, a cesariana implica a maior permanência hospitalar ${ }^{15}$.

O risco de infecção puerperal em pacientes submetidos à cesárea eletiva é três vezes mais elevada que às submetidas parto vaginal. Ressalta-se que os principais fatores de risco são: ruptura prematura das membranas ovulares e/ou trabalho de parto prolongados; manipulação vaginal excessiva (toques); más condições de assepsia; debilidade imunológica; desnutrição ou obesidade; traumas cirúrgicos; cesarianas desnecessárias e retenção de restos ovulares ${ }^{14}$.

A presença de infecção pós-parto, independentemente do sítio de infecção, ou infecção da ferida operatória, é maior nas cesáreas, bem como a necessidade de internação em UTI. O risco de hemorragia, histerectomia e transfusão de sangue ${ }^{6}$.

A infecção puerperal está relacionada com as infecções da assistência em saúde, sendo uma complicação do período gravídico puerperal, a qual contribui para o aumento da morbimortalidade materna. Assim, a infecção puerperal é compreendida como qualquer infecção bacteriana do trato genital feminino concorrente ao processo do parto e nascimento ${ }^{14}$. Os riscos para o feto e recém-nascidos também devem ser considerados durante o processo de escolha da via de parto na ausência de indicação médica para a cesárea. Pois cesárea a pedido sem indicação médica aumenta o risco de complicações respiratórias no recém-nascido. Aumento nas taxas de cesárea também está associado a maiores taxas de mortalidade fetal e um maior número de bebês admitidos em UTI neonatal por sete dias ou mais, mesmo após controle para prematuridade ${ }^{6}$.

Apesar do avanço científico e tecnológico nas diversas áreas do conhecimento, a infecção puerperal constitui, ainda, um grande problema de saúde pública, evidenciado pela sua alta prevalência de morbidade e letalidade ${ }^{14}$.

\section{CONSIDERAÇÕES FINAIS}

Concui-se que a disponibilidade de informações sobre gestação e parto favorece a inclusão das gestantes no processo decisório, oportunizando a manifestação de sua autonomia em relação à via de parto.

A vivência do parto normal é um desafio para as mulheres, mas que os sentimentos positivos descritos superam essas dificuldades. De outro modo, a cesárea, apesar de ser mais rápida e 
cômoda, ao propiciar um momento agradável e tranquilo, não está associada a sentimentos positivos, mas, sim, aos benefícios físicos de sua realização.

O principal desafio relacionado às cesarianas está em fazer o melhor uso desse procedimento que, por um lado, é um recurso importante para a redução da mortalidade materna e neonatal, mas por outro, quando usado de maneira excessiva, pode estar associado a um risco aumentado de resultados maternos graves.

É importante ressaltar que, para o bem-estar materno e fetal, as representações sociais do parto normal e da cesárea incluem aspectos físicos, emocionais e socioculturais envolvidos, que precisam ser respeitados na individualidade e integralidade de cada ser humano, como a preferência por uma ou outra via de parto.

Vale ressaltar que a cesárea oferece maior risco de complicações comparadas ao parto vaginal. Contudo, cabe ao enfermeiro utilizar cuidados adequado à mulher após a cesárea que muitas das vezes é indicada para contornar problemas que surgem durante o pré-natal e na evolução de um parto vaginal.

Esta revisão pode fornecer aos profissionais da área da saúde que atendem diretamente as gestantes e parturientes, informações que facilitem o entendimento de expressões psicológicas como o medo, a insegurança e a fragilidade que este processo oferece a mulher, além da possibilidade de conhecer previamente alguns fatores que interferem na decisão destas por determinado tipo de parto. Por sua vez serviram de instrumento para a melhora na qualidade da atenção desempenhada no ciclo gravídico-puerperal, tornando-o mais humanizado.

\section{REFERÊNCIAS}

1. Pereira SS, Oliveira ICMS, Santos JBS, Carvalho MCDMP. Parto natural: a atuação do enfermeiro diante da assistência humanizada. Tempus Actas de Saúde Coletiva [Internet]. 2016 [citado em 10 mai 2018]; 10(3), 199-213. Disponível em: $<$ http://www.tempusactas.unb.br/index.php/tempus/article/view/1727>.

2. Velho MB, Santos EKA, Collaço VS. Parto normal e cesárea: representações sociais de mulheres que os vivenciaram. Revista Brasileira de Enfermagem [Internet]. 2014 [citado em 10 mai 2018]; 67(2). Disponível em: <http://www.redalyc.org/html/2670/267030687017/>.

3. Rech C, Leidemer V, Rodrigues AF, Fritsch E. Parto normal, natural ou cesariana, uma questão de escolha? Mostra IFTec em Resumos [Internet]. 2018 [citado em 10 mai 2018]; (5). Disponível em: <https://periodicos.ifrs.edu.br/index.php/MostraIFTec/article/view/2976>. 
4. Oliveira RR, Melo EC, Novaes ES, Ferracioli PLRV, Mathias TAF. Fatores associados ao parto cesárea nos sistemas público e privado de atenção à saúde. Revista da Escola de Enfermagem da USP [Internet]. 2016 [citado em 10 mai 2018]; 50(5), 734-741. Disponível em: $<$ http://www.redalyc.org/articulo.oa?id=361048760004 $>$.

5. Minuzzi A, Rezende CL. Fatores de influência na escolha da via de parto: uma revisão de literatura. Ver Uningá [Internet]. 2017 [citado em 10 mai 2018]; 14(1), 11-11. Disponível em: $<\mathrm{http}$ //revista.uninga.br/index.php/uningareviews/article/view/712/359>.

6. Mascarello KC, Horta BL, Silveira MF. Complicações maternas e cesárea sem indicação: revisão sistemática e meta-análise. Revista de Saúde Pública [Internet]. 2017 [citado em 10 mai 2018]; 51, 1-12. Disponível em: <http://www.redalyc.org/pdf/672/67249591107.pdf>.

7. Cesar JA, Sauer JP, Carlotto K, Montagner ME, Mendoza-Sassi RA. Cesariana sob demanda: um estudo de base populacional no sul do Brasil. Revista Brasileira de Saúde Materno Infantil [Internet]. 2017 [citado em 10 mai 2018]; 17(1), 99-105. Disponível em: $<$ http://www.scielo.br/pdf/rbsmi/v17n1/pt_1519-3829-rbsmi-17-01-0099.pdf>.

8. Ramos ALL, Benício FCM, Silva RKN, Costa VRR, Mendes IC. Conhecimento de puérpuras quanto aos cuidados maternos pós-cesariana e atenção ao recém-nascido. Mostra Interdisciplinar do curso de Enfermagem [Internet]. 2017 [citado em 10 mai 2018]; 2(2). Disponível em: <http://publicacoesacademicas.fcrs.edu.br/index.php/mice/article/view/1146>.

9. Domingues RMSM, Dias MAB, Nakamura-Pereira M, Torres JA, d'Orsi E, Pereira APE, et al. Processo de decisão pelo tipo de parto no Brasil: da preferência inicial das mulheres à via de parto final.Cadernos de Saúde Pública [Internet]. 2014 [citado em 10 mai 2018; 30, S101S116. Disponível em: <https://www.scielosp.org/article/csp/2014.v30suppl1/S101-S116/pt/>.

10. Silva IA, Andrade ÉWOF, Morais FF, Silva RSDS, Oliveira LS. Percepção das puérperas acerca da assistência de enfermagem no parto humanizado. Rer Uningá [Internet]. 2018 [citado em 10 mai 2018]; 53(2). Disponível em: $<$ http://revista.uninga.br/index.php/uninga/article/view/1440>.

11. Oliveira ALG, Nascimento PDS, Sales PAP, Soares R, Moreira JP. Assistência do enfermeiro à parturiente: foco no parto humanizado. Revista de trabalhos acadêmicosuniverso campos dos Goytacazes [Internet]. 2017 [citado em 01 out 2018]; 1(8). Disponível em: $\quad<$ http://www.revista.universo.edu.br/index.php?journal=1CAMPOSDOSGOYTACAZ ES2\&page=article\&op=view\&path $\% 5 \mathrm{~B} \% 5 \mathrm{D}=4487$

12. Almeida OSC, Gama ER, Bahiana PM. Humanização do parto: a atuação dos enfermeiros. Revista Enfermagem Contemporânea [Internet]. 2015 [citado em 01 out 2018]; 4(1). Disponível em: <https://www5.bahiana.edu.br/index.php/enfermagem/article /view/456/437>. 
13. Nascimento RRP, Arantes SL, Souza EDC, Contrera L, Sales APA. Escolha do tipo de parto: fatores relatados por puérperas. Revista Gaúcha de Enfermagem [Internet]. 2015 [citado em 01 out 2018];36, 119-126. Disponível em: <http://www.seer.ufrgs.br /RevistaGauchadeEnfermagem/article/view/56496>.

14. Duarte MR, Chrizostimo MM, Christovam BP, Ferreira SCM, Souza DF, Rodrigues DP. Atuação do enfermeiro no controle de infecção puerperal: revisão integrativa. Revista de enfermagem UFPE [Internet]. 2014 [citado em 01 out 2018]; 8(2), 433-441. Disponível em: $<$ https://periodicos.ufpe.br/revistas/revistaenfermagem/article/view/9691>.

15. Entringer AP, Pinto M, Dias MAB, Gomes MADSM. Análise de custo-efetividade do parto vaginal espontâneo e da cesariana eletiva para gestantes de risco habitual no Sistema Único de Saúde. Cadernos de Saúde Pública [Internet]; 2018 [citado em 01 out 2018]; 34, e00022517. Disponível em: <https://www.scielosp.org/article/csp/2018.v34n5/e00022517/>. 